\title{
Shot noise of Coulomb drag current
}

\author{
V. L. Gurevich and M. I. Muradov \\ Solid State Physics Department, A. F. Ioffe Institute, 194021 Saint Petersburg, Russia
}

(November 21, 2018)

\begin{abstract}
We work out a theory of shot noise in a special case. This is a noise of the Coulomb drag current excited under the ballistic transport regime in a onedimensional nanowire by a ballistic non-Ohmic current in a nearby parallel nanowire. We predict sharp oscillation of the noise power as a function of gate voltage or the chemical potential of electrons. We also study dependence of the noise on the voltage $V$ across the driving wire. For relatively large values of $V$ the spectral density of the low-frequency noise is proportional to $V^{2}$.
\end{abstract}

\section{INTRODUCTION}

The purpose of the present paper is to study the shot noise of the Coulomb drag current in the course of ballistic (collisionless) electron transport in a quantum nanowire due to a ballistic driving current in an adjacent nanowire. The possibility of the Coulomb drag effect in the ballistic regime has been demonstrated by Gurevich, Pevzner and Fenton [1] for an Ohmic regime and by Gurevich and Muradov [2] for a non-Ohmic one and has been experimentally observed by Debray et al. [3].

If two wires, 1 and 2, are close and are parallel, the drag force due to the ballistic current in wire 2 acts as a sort of permanent acceleration on the electrons of wire 1 via the Coulomb interaction. As a result, there appears a current noise in wire 1 which depends on the voltage $V$ across wire 2. Such a voltage-dependent noise can be looked upon as a sort of shot noise. It is a theory of this noise that we will consider in the present paper.

Let the two wires be much shorter than the electron mean free path (typically a few $\mu \mathrm{m}$ ). Such nanoscale systems are characterized by low electron densities, which may be varied by 
means of the gate voltage. As in Ref. [1], we assume the wires to be of different widths though having the same lengths $L$. Like in Refs. [1,2] we consider the interaction processes when electrons in nanowires 1 and 2 after scattering remain within the initial subbands having the dispersion laws

$$
\varepsilon_{n p}^{(1)}=\varepsilon_{n}^{(1)}(0)+p^{2} / 2 m, \quad \varepsilon_{n^{\prime} p}^{(2)}=\varepsilon_{n^{\prime}}^{(2)}(0)+p^{2} / 2 m,
$$

where $p$ is the $x$-component of the electron quasimomentum, the $x$ axis is parallel to the wires while $n$ and $n^{\prime}$ are the subbands' numbers.

The Boltzmann equation for fluctuations of the distribution function describing electrons of wire 1 is

$$
\left(\frac{\partial}{\partial t}+v \frac{\partial}{\partial x}\right) \delta F_{n p}^{(1)}=-J_{p} \delta F_{n p}^{(1)}+y_{n p}^{(1)}
$$

where $\delta F^{(1)}$ are the fluctuations of the electron distribution function in wire 1 , and $y_{n p}^{(1)}$ is the Langevin random force originating in the interwire electron-electron scattering. It is only such a scattering that we will take into account in this otherwise ballistic system.

As in Refs. [1],2], we will solve Eq. (2) by iterations. The collision term $J_{p}$ has the following form

$$
\begin{aligned}
& J_{p} \Psi_{n p} \\
& =\Psi_{n p} \sum_{n^{\prime} p^{\prime} q} W_{1 p n, 2 p^{\prime} n^{\prime}}^{1 p+q n, 2 p^{\prime}-q n^{\prime}}\left[F_{n^{\prime} p^{\prime}}^{(2)}\left(1-F_{n p+q}^{(1)}\right)\left(1-F_{n^{\prime} p^{\prime}-q}^{(2)}\right)+\left(1-F_{n^{\prime} p^{\prime}}^{(2)}\right) F_{n p+q}^{(1)} F_{n^{\prime} p^{\prime}-q}^{(2)}\right] \\
& -\sum_{n^{\prime} p^{\prime} q} \Psi_{n p+q} W_{1 p n, 2 p^{\prime} n^{\prime}}^{1 p+q n, 2 p^{\prime}-q n^{\prime}}\left[F_{n^{\prime} p^{\prime}-q}^{(2)}\left(1-F_{n p}^{(1)}\right)\left(1-F_{n^{\prime} p^{\prime}}^{(2)}\right)+\left(1-F_{n^{\prime} p^{\prime}-q}^{(2)}\right) F_{n p}^{(1)} F_{n^{\prime} p^{\prime}}^{(2)}\right] .
\end{aligned}
$$

Here and henceforth it is implied that summation over $p$ or $p^{\prime}$ includes also the spin summation.

Applying the method of iterations we insert into the right-hand side of Eq. (2) $\delta F_{n p}^{(1)}$ in the zeroth approximation given by the second and the third terms on the right-hand side of Eq. (8) — see below. We do not take into account $\delta F_{n p}^{(2)}$ as in this approximation the functions $\delta F_{n p}^{(1)}$ and $\delta F_{n p}^{(2)}$ are uncorrelated being emitted by different reservoirs. 
The correlation function of the Langevin forces can be obtained using the procedure described in [4]

$$
\begin{array}{r}
\left\langle y_{n p}^{(1)}(\mathbf{r}) y_{n p^{\prime}}^{(1)}\left(\mathbf{r}^{\prime}\right)\right\rangle_{\omega}=\delta_{\mathbf{r r}^{\prime}} \sum_{n^{\prime} p_{1} q}\left(\delta_{p p^{\prime}}-\delta_{q, p^{\prime}-p}\right) W_{1 p n, 2 p_{1} n^{\prime}}^{1 p+q n, 2 p_{1}-q n^{\prime}} \\
\times\left[F_{n p}^{(1)} F_{n^{\prime} p_{1}}^{(2)}\left(1-F_{n p+q}^{(1)}\right)\left(1-F_{n^{\prime} p_{1}-q}^{(2)}\right)+F_{n p+q}^{(1)} F_{n^{\prime} p_{1}-q}^{(2)}\left(1-F_{n p}^{(1)}\right)\left(1-F_{n^{\prime} p_{1}}^{(2)}\right)\right] .
\end{array}
$$

Here the first term on the right-hand side, including $\delta_{p p^{\prime}}$, is a sum of the 'in' and 'out' terms in the Boltzmann equation for the state $n p$, whereas the other term is the sum of collision probabilities where one of the initial states is $n p$ and one of the final states is $n p^{\prime}$ et vice versa. The correlation function, as well as the scattering integral, satisfy the following relations representing the particle number conservation

$$
\sum_{n p}\left\langle y_{n p}^{(1)}(\mathbf{r}) y_{n p^{\prime}}^{(1)}\left(\mathbf{r}^{\prime}\right)\right\rangle_{\omega}=\sum_{n p^{\prime}}\left\langle y_{n p}^{(1)}(\mathbf{r}) y_{n p^{\prime}}^{(1)}\left(\mathbf{r}^{\prime}\right)\right\rangle_{\omega}=0, \quad \sum_{p} J_{p} \Psi_{n p}=0 .
$$

The solution of Eq.(2) can be written using the Green function satisfying the equation

$$
\left(\frac{\partial}{\partial t}+v \frac{\partial}{\partial x}\right) G\left(x t \mid x^{\prime} t^{\prime}\right)=\delta\left(x-x^{\prime}\right) \delta\left(t-t^{\prime}\right) .
$$

We have

$$
G\left(x t \mid x^{\prime} t^{\prime}\right)=\frac{1}{|v|} \delta\left(t-t^{\prime}-\frac{x-x^{\prime}}{v}\right) \theta\left(\frac{x-x^{\prime}}{v}\right),
$$

where $v=p / m$ is the $x$-component of the electron velocity, so that

$$
\begin{array}{r}
\delta F_{n p}(x, t)=\int_{-\infty}^{+\infty} d t^{\prime} d x^{\prime} G\left(x t \mid x^{\prime} t^{\prime}\right)\left\{-J_{p} \delta F_{n p}+y_{n p}\left(x^{\prime}, t^{\prime}\right)\right\} \\
+|v| \int_{-\infty}^{+\infty} d t^{\prime} G\left(x t \mid-\frac{L}{2}, t^{\prime}\right) \delta F_{n p}\left(-\frac{L}{2}, t^{\prime}\right)+|v| \int_{-\infty}^{+\infty} d t^{\prime} G\left(x t \mid \frac{L}{2}, t^{\prime}\right) \delta F_{n p}\left(\frac{L}{2}, t^{\prime}\right) .
\end{array}
$$

For the current fluctuations we get

$$
\delta I(x, t)=\frac{e}{L} \sum_{n p} v \delta F_{n p}(x, t)
$$

The collision integral takes into account only the interwire electron-electron scattering.

Using the particle number conserving property (5) we write 


$$
\begin{aligned}
\delta I(x, t) & =\frac{e}{L} \sum_{n p>0} \int_{-L / 2}^{L / 2} d x^{\prime}\left\{-J_{p} \delta F_{n p}\left(x^{\prime}, t-\frac{x-x^{\prime}}{v}\right)+y_{n p}\left(x^{\prime}, t-\frac{x-x^{\prime}}{v}\right)\right\} \\
& +\frac{e}{L} \sum_{n p>0} v \delta F_{n p}\left(-\frac{L}{2}, t-\frac{x+L / 2}{v}\right)+\frac{e}{L} \sum_{n p<0} v \delta F_{n p}\left(\frac{L}{2}, t-\frac{x-L / 2}{v}\right) .
\end{aligned}
$$

Here the first term on the right-hand side describes the noise induced by the collisions of the electrons in wire 1 with the electrons in wire 2 while the rest two terms describe the contributions of fluctuations of distributions for the electrons entering wire 1 from the left and the right boundaries. Accordingly, the noise power consists of the Nyquist noise and the nonequilibrium noise due to collisions with the nonequilibrium electrons in wire 2 .

Using the identity

$$
\left\langle\delta F_{n p}(x, t) \delta F_{n p^{\prime}}\left(x^{\prime}, t^{\prime}\right)\right\rangle=L \delta_{p p^{\prime}} F_{n p}\left(1-F_{n p}\right) \delta\left[x-x^{\prime}-v_{p}\left(t-t^{\prime}\right)\right]
$$

we have for the total power of the low frequency noise $P_{\text {tot }}=P_{\mathrm{N}}+P$ where the power of the equilibrium Nyquist noise is

$$
P_{\mathrm{N}}=\frac{e^{2}}{\pi \hbar} \sum_{n} \int_{0}^{\infty} d p v\left\{f_{L}\left(\varepsilon_{n p}-\mu\right)\left[1-f_{L}\left(\varepsilon_{n p}-\mu\right)\right]+f_{R}\left(\varepsilon_{n p}-\mu\right)\left[1-f_{R}\left(\varepsilon_{n p}-\mu\right)\right]\right\},
$$

or

$$
P_{\mathrm{N}}=4 G T, \quad G=e^{2} / \pi \hbar N
$$

Here $N$ is the number of open channels in wire 2. We will write throughout the paper $T$ instead of $k_{\mathrm{B}} T$ where $T$ is the temperature. The noise power due to the drag is

$$
P=P_{S}+P_{L}+P_{R}
$$

where

$$
P_{S}=2 e^{2} \sum_{n, p>0, p^{\prime}>0}<y_{n p} y_{n p^{\prime}}>_{\omega}
$$

describes fluctuations induced by the sources in the wire,

$$
P_{L}=-4 e^{2} \sum_{n, p^{\prime}>0} \sum_{p>0} F_{n p}^{(1)}\left(1-F_{n p}^{(1)}\right) J_{p^{\prime}} \delta_{p p^{\prime}}
$$


describes the scattering of fluctuations entering the wire from the left reservoir, and

$$
P_{R}=4 e^{2} \sum_{n, p^{\prime}>0} \sum_{p<0} F_{n p}^{(1)}\left(1-F_{n p}^{(1)}\right) J_{p^{\prime}} \delta_{p p^{\prime}}
$$

describes the scattering of fluctuations entering the wire from the right reservoir.

The scattering probability is

$$
W_{1 p n, 2 p^{\prime} n^{\prime}}^{1 p+q n, 2 p^{\prime}-q n^{\prime}}=\frac{2 \pi}{\hbar}\left|V_{1 p n, 2 p^{\prime} n^{\prime}}^{1 p+q n, 2 p^{\prime}-q n^{\prime}}\right|^{2} \delta\left(\varepsilon_{n p}^{(1)}+\varepsilon_{n^{\prime} p^{\prime}}^{(2)}-\varepsilon_{n p+q}^{(1)}-\varepsilon_{n^{\prime} p^{\prime}-q}^{(2)}\right) .
$$

It can be transformed with the help of relations (see Ref. [1])

$$
\delta\left(\varepsilon_{n p}^{(1)}+\varepsilon_{n^{\prime} p^{\prime}}^{(2)}-\varepsilon_{n p+q}^{(1)}-\varepsilon_{n^{\prime} p^{\prime}-q}^{(2)}\right)=\frac{m}{\left|p-p^{\prime}\right|} \delta\left(q+p-p^{\prime}\right)
$$

and

$$
\left|\left\langle n p-q, n^{\prime} p|V| n p, n^{\prime} p-q\right\rangle\right|^{2}=\left(\frac{2 e^{2}}{\kappa L}\right)^{2} g_{n n^{\prime}}(q)
$$

where

$$
g_{n n^{\prime}}(q)=\left(\int d \mathbf{r}_{\perp} \int d \mathbf{r}_{\perp}^{\prime}\left|\phi_{n}\left(\mathbf{r}_{\perp}\right)\right|^{2} K_{0}\left(|q| \hbar^{-1}\left|\mathbf{r}_{\perp}-\mathbf{r}_{\perp}^{\prime}\right|\right)\left|\phi_{n^{\prime}}\left(\mathbf{r}_{\perp}^{\prime}\right)\right|^{2}\right)^{2},
$$

$\phi_{n}\left(\mathbf{r}_{\perp}\right)$ being the functions of transverse quantization. We assume, in the spirit of the Landauer-Büttiker-Imry [7] approach, wire 1 to be connected to reservoirs which we call 'left' $(l)$ and 'right' $(r)$, each of these being in independent equilibrium described by the shifted chemical potentials $\mu^{(l)}=\mu-\Delta \mu / 2$ and $\mu^{(r)}=\mu+\Delta \mu / 2$. Here $\mu$ is the average chemical potential while $\Delta \mu / e=V$ is the voltage across wire 2 (we will assume that $e V>0$ ) and $e<0$ is the electron charge. Therefore, the electrons entering the wire from the 'left' ('right') and having quasimomenta $p^{\prime}>0\left[p^{\prime}<0\right]$ are described by $F_{n^{\prime} p^{\prime}}^{(2)}=f\left(\varepsilon_{n^{\prime} p^{\prime}}^{(2)}-\mu^{(l)}\right)$ $\left[F_{n^{\prime} p^{\prime}}^{(2)}=f\left(\varepsilon_{n^{\prime} p^{\prime}}^{(2)}-\mu^{(r)}\right)\right]$ respectively. For the noise power $P_{S}$ we get

$$
P_{S}=2 e^{2} m \frac{2 \pi}{\hbar}\left(\frac{L}{2 \pi \hbar}\right)\left(\frac{2 e^{2}}{\kappa L}\right)^{2}\left(\frac{2 L}{2 \pi \hbar}\right)^{2} \sum_{n n^{\prime}} \int_{0}^{\infty} d p \int_{0}^{\infty} d p^{\prime} \frac{g_{n n^{\prime}}\left(p+p^{\prime}\right)}{p+p^{\prime}} \mathcal{S}
$$

where 


$$
\begin{aligned}
\mathcal{S} & =f\left(\varepsilon_{n p^{\prime}}-\mu\right)\left[1-f\left(\varepsilon_{n^{\prime} p^{\prime}}-\mu-\frac{e V}{2}\right)\right] \\
& \times f\left(\varepsilon_{n^{\prime} p}-\mu+\frac{e V}{2}\right)\left[1-f\left(\varepsilon_{n p}-\mu\right)\right]\left(1+\exp \frac{e V}{T}\right)
\end{aligned}
$$

One can take out of the integral all of the slowly varying functions. Exploiting the relation

$$
\int_{-\infty}^{+\infty} d x \frac{\exp (x+2 a)}{[1+\exp (x+2 a)][1+\exp (x+2 b)]}=(a-b) \frac{\exp (a-b)}{\sinh (a-b)}
$$

one gets

$$
P_{S}=-2 e J \operatorname{coth}\left(\frac{e V}{2 T}\right)
$$

The drag current $J$ is according to Ref. [2]

$$
J=\frac{1}{2} J_{0} \sinh x^{(0)} \cdot \frac{x_{n n^{\prime}}^{(-)}}{\sinh x_{n n^{\prime}}^{(-)}} \cdot \frac{x_{n n^{\prime}}^{(+)}}{\sinh x_{n n^{\prime}}^{(+)}} .
$$

Here we have introduced notation $x^{(0)}=e V / 2 T, x_{n n^{\prime}}^{( \pm)}=e V / 4 T \pm \varepsilon_{n n^{\prime}} / 2 T$,

$$
J_{0}=-\frac{8 e^{5} m^{3} L T^{2}}{\kappa^{2} \pi^{2} \hbar^{4}} \cdot \frac{g_{n n^{\prime}}\left(2 p_{n}\right)}{p_{n}^{3}}
$$

(where $\kappa$ is the dielectric susceptibility) and

$$
\varepsilon_{n n^{\prime}}=\varepsilon_{n}^{(1)}(0)-\varepsilon_{n^{\prime}}^{(2)}(0), \quad m v_{n}=p_{n}=\sqrt{2 m\left[\mu-\varepsilon_{n}^{(1)}(0)\right]} .
$$

According to Refs. [1, 2], the current $J$ as a function of the gate voltage comprises a system of spikes; the position of each spike is determined by a coincidence of a pair of levels of transverse quantization, $\varepsilon_{n}(0)$ and $\varepsilon_{n^{\prime}}(0)$ in both wires. Using the explicit form of the scattering operator Eq.(3) one can show that the noise power $P_{R}$ can be expressed through $P_{L}$ simply by the replacement $e V \rightarrow-e V$. Indeed, taking into consideration that summation in (15) is performed over the positive quasimomenta $p>0$ and using the expression for the scattering probability (17), (18) we get

$$
\begin{gathered}
\sum_{p^{\prime}>0} J_{p^{\prime}} \delta_{p p^{\prime}}=\left(\frac{2 e^{2}}{\kappa L}\right)^{2} m \frac{L}{2 \pi \hbar} \sum_{n^{\prime}, p^{\prime}<0} \frac{g_{n n^{\prime}}\left(p^{\prime}-p\right)}{\left|p-p^{\prime}\right|} \mathcal{F}, \\
\mathcal{F}=\left[F_{n^{\prime} p^{\prime}}^{(2)}\left(1-F_{n p^{\prime}}^{(1)}\right)\left(1-F_{n^{\prime} p}^{(2)}\right)+\left(1-F_{n^{\prime} p^{\prime}}^{(2)}\right) F_{n p^{\prime}}^{(1)} F_{n^{\prime} p}^{(2)}\right]
\end{gathered}
$$


in the expression for $P_{L}$ while in the expression for $P_{R}$ (taking into account that there the sum is over the negative $p$ ) we have

$$
\sum_{p^{\prime}>0} J_{p^{\prime}} \delta_{p p^{\prime}}=-\left(\frac{2 e^{2}}{\kappa L}\right)^{2} m \frac{L}{2 \pi \hbar} \sum_{n^{\prime}, p^{\prime}>0} \frac{g_{n n^{\prime}}\left(p^{\prime}-p\right)}{\left|p-p^{\prime}\right|} \mathcal{F}
$$

Now replacing $F_{s k}^{(2)}$ by $f\left(\varepsilon_{s k}-\mu+e V / 2\right)$ and $f\left(\varepsilon_{s k}-\mu-e V / 2\right)$ for $p>0$ and $p<0$ respectively, for the $P_{L}$ we have

$$
P_{L}=-4 e^{2} m \frac{2 \pi}{\hbar}\left(\frac{L}{2 \pi \hbar}\right)\left(\frac{2 e^{2}}{\kappa L}\right)^{2}\left(\frac{2 L}{2 \pi \hbar}\right)^{2} \sum_{n n^{\prime}} \int_{0}^{\infty} d p \int_{0}^{\infty} d p^{\prime} \frac{g_{n n^{\prime}}\left(p+p^{\prime}\right)}{p+p^{\prime}} \mathcal{L}
$$

where

$$
\begin{aligned}
\mathcal{L} & =f\left(\varepsilon_{n p}-\mu\right)\left[1-f\left(\varepsilon_{n p}-\mu\right)\right] \\
& \times\left\{f\left(\varepsilon_{n^{\prime} p}-\mu+\frac{e V}{2}\right) f\left(\varepsilon_{n p^{\prime}}-\mu\right)\left[1-f\left(\varepsilon_{n^{\prime} p^{\prime}}-\mu-\frac{e V}{2}\right)\right]\right. \\
& \left.+f\left(\varepsilon_{n^{\prime} p^{\prime}}-\mu-\frac{e V}{2}\right)\left[1-f\left(\varepsilon_{n p^{\prime}}-\mu\right)\right]\left[1-f\left(\varepsilon_{n^{\prime} p}-\mu+\frac{e V}{2}\right)\right]\right\}
\end{aligned}
$$

Finally we get

$$
P_{L}=2 e J\left[\frac{1}{x_{n n^{\prime}}^{(-)}}-\frac{1}{\sinh x^{(0)}} \cdot \frac{\sinh x_{n n^{\prime}}^{(+)}}{\sinh x_{n n^{\prime}}^{(-)}}\right]
$$

The sum of $P_{L}$ and $P_{R}$ is

$$
P_{L}+P_{R}=-2 e J\left\{\frac{1}{\sinh x^{(0)}}\left[\frac{\sinh x_{n n^{\prime}}^{(+)}}{\sinh x_{n n^{\prime}}^{(-)}}+\frac{\sinh x_{n n^{\prime}}^{(-)}}{\sinh x_{n n^{\prime}}^{(+)}}\right]-\frac{x^{(0)}}{x_{n n^{\prime}}^{(-)} x_{n n^{\prime}}^{(+)}}\right\}
$$

where $J$ is given by Eq. (25). For $e V \ll T$ one gets for the variation of the total noise power in wire 1 due to the presence of wire 2

$$
P=P_{S}+P_{L}+P_{R}=e J_{0}\left[\frac{\varepsilon_{n n^{\prime}}}{2 T}\right]^{2} \cdot\left[\sinh \left(\frac{\varepsilon_{n n^{\prime}}}{2 T}\right)\right]^{-2}
$$

One can verify that this result is consistent with the fluctuation-dissipation theorem. Indeed, the linear response to the voltage $V^{(1)}$ across wire 1 in this case can be written as

$$
J=\left(G-G_{\mathrm{tr}}\right) V^{(1)}
$$

Here, according to Eq.(25), we have introduced the transconductance $G_{t r}$ 


$$
G_{\mathrm{tr}}=-\frac{e}{4 T} J_{0}\left[\frac{\varepsilon_{n n^{\prime}}}{2 T}\right]^{2}\left[\sinh \left(\frac{\varepsilon_{n n^{\prime}}}{2 T}\right)\right]^{-2} .
$$

We assume that $J, V^{(1)}$ (and, of course, $G_{\mathrm{tr}}$ ) are positive quantities; then the fluctuationdissipation theorem states that the additional equilibrium contribution to the noise due to the wire 2 is

$$
P=-4 G_{\mathrm{tr}} T
$$

which coincides with Eq. (35).

Let us consider in detail the opposite case $e V \gg T$. In this case one gets a nonvanishing result for Eq.(25) only if $\left|\varepsilon_{n n \prime}\right|<e V / 2$ and one obtains the Poisson limit for the noise power

$$
P=-2 e J
$$

where the drag current is given by

$$
J=\mathcal{B}\left[\left(\frac{e V}{2}\right)^{2}-\left(\varepsilon_{n n^{\prime}}\right)^{2}\right], \quad \mathcal{B}=-\frac{2 e^{5} m^{3} L}{\kappa^{2} \pi^{2} \hbar^{4}} \cdot \frac{g_{n n^{\prime}}\left(2 p_{n}\right)}{p_{n}^{3}} .
$$

The situation is illustrated in Figure 1, where we plot the noise power versus applied driving voltage for various values of the difference of transverse energy levels $\varepsilon_{n n^{\prime}}$. For the small values of $\mathrm{eV} / T$ we have an additional contribution to the thermal (equilibrium) noise due to wire 2 described by Eq. (35), while for the large values of $e V / T$ the contribution to the (now nonequilibrium) noise in the drag wire is described by a quadratic dependence of Eq.(40). To clear the situation further we plot the dependence of the noise power on the difference of transverse energy levels $\varepsilon_{n n^{\prime}}$ in Figure 2.

In summary, we have developed a theory of a shot noise in a quantum wire excited by a non-Ohmic current in a nearby parallel nanowire. A ballistic transport in both nanowires is assumed. The shot noise power $P$ as a function of the gate voltage comprises a system of spikes; the position of each spike is determined by a coincidence of a pair of levels of transverse quantization, $\varepsilon_{n}^{(1)}(0)$ and $\varepsilon_{n^{\prime}}^{(2)}(0)$ in both wires. For $e V \gg T, P$ is a quadratic function of the driving voltage $V$. The effect may play an important role in the investigation of the interwire Coulomb scattering as well as 1D band structure of the wires. 
The authors are grateful to P. Debray for sending them a preprint of paper [3] prior to publication. The authors are pleased to acknowledge the support for this work by the Russian National Fund of Fundamental Research (Grant \# 97-02-18286-a).

[1] V. L. Gurevich, V. B. Pevzner, and E. W. Fenton, J. Phys.: Condens. Matter 10, 2551 (1998)

[2] V. L. Gurevich, M. I. Muradov, to be published.

[3] P. Debray, P. Vasilopulos, O. Raichev, R. Perrin, M. Rahman and W. C. Mitchel, preprint.

[4] S. V. Gantsevich, V. L. Gurevich, and R. Katilius, Fiz. Tverd. Tela 11, 308 (1969) [Sov. Phys. Sol. State 11, 247 (1969)]; Zh. Exsp. Teor. Fiz. 57, 503 (1969) [Sov.Phys. - JETP 30, 276 (1970)].

[5] Sh. M. Kogan and A. Ya. Shul'man, Zh. Exsp. Teor. Fiz. 56, 862 (1969) [Sov.Phys. - JETP 29, $467(1969)]$.

[6] M. I. Muradov, Phys. Rev. B 58, 12883 (1998).

[7] Y. Imry, Directions in Condensed Matter Physics, ed. G. Grinstein and G. Mazenko, 1986 (Singapore: World Scientific), 101; M. Büttiker, Phys.Rev. Lett., 57, 1761 (1986). 


\section{FIGURE CAPTIONS}

1. Fig. 1. Noise power $P$ in wire 1 as a function of the voltage $V$ applied across wire 2

for $\varepsilon_{n n^{\prime}} / T=0,1,2,3$. As $\varepsilon_{n n^{\prime}} / T$ goes up the curves are shifted to the right in the upper part of the figure.

2. Fig. 2. Noise power $P$ as a function of $\varepsilon_{n n^{\prime}} / T$ for $e V / T=0,3,6,9,12$. The values of the functions for $\varepsilon_{n n^{\prime}}=0$ go up as $e V / T$ goes up. 